\title{
Effect of Antistatic Additives on Mechanical and Electrical Properties of Polyethylene Foams
}

\author{
J. C. Costa, ${ }^{1,2}$ M. Oliveira, ${ }^{2}$ A. V. Machado, ${ }^{2}$ S. Lanceros-Méndez, ${ }^{3}$ G. Botelho ${ }^{4}$ \\ ${ }^{1}$ Epoli-Espumas de polietileno, S.A., Apartado 2106, 4471-908 Avioso (Sta. Maria), Portugal \\ ${ }^{2}$ Departamento de Engenharia de Polímeros, Universidade do Minho, Campus de Azurém, \\ 4800-058 Guimarães, Portugal \\ ${ }^{3}$ Departamento de Física, Universidade do Minho, 4710-057 Braga, Portugal \\ ${ }^{4}$ Departamento de Química, Centro de Química, Universidade do Minho, Campus de Gualtar, \\ 4710-057 Braga, Portugal
}

Received 4 March 2008; accepted 8 October 2008

DOI 10.1002/app.29503

Published online 9 February 2009 in Wiley InterScience (www.interscience.wiley.com).

\begin{abstract}
Polyethylene foams with different antistatic additives (Atmer 7325, Atmer 7105, and Atmer 190) were prepared by extrusion and stored during 6 months of time span in a real life environment. The antistatic performance was evaluated by measuring the surface resistivity and throughout decay-time experiments. Mechanical properties and migration tests were also performed. It was found that the migration of antistatic agents is in general low enough, which allows to maintain the antistatic performance for periods of time larger than 6 months. The tests revealed that the desired low surface resistance and required low static decay time
\end{abstract}

could be achieved with all the antistatic agents under test. Moreover, the additives with amina (Atmer 7105 and 7325) as an active agent showed slightly better antistatic performance than the one with the ionic agent (Atmer 190). The addition of an antistatic agent does not significantly affect the mechanical behavior of the foam indicating a desired feature concerning industrial applications. ( 2009 Wiley Periodicals, Inc. J Appl Polym Sci 112: 15951600,2009

Key words: polyethylene foam; antistatic additives; electrical properties; mechanical performance

\section{INTRODUCTION}

Many polymer materials, in their intrinsic form, do not have the surface properties required for incorporation into commercial products such as packaging, conductors, sensors, and active electrodes. Polymers, in general, combine high surface resistivity with a low dielectric constant, ${ }^{1}$ which, together with the rough handling that plastics often receive during transport and processing, may lead to high static charges, to $15,000 \mathrm{~V} / \mathrm{cm}$ in air. This fact increases handling problems during transport and storage as well as packing dust contamination. It affects both appearance and performance of end-products, increases the risk of electrical shocks and the risk of electrical discharge causing fire or explosion. The aforementioned characteristics and risks are mainly due to electrical insulation effect of polymers and to their low surface energy, the presence of surface contaminants and weak boundary layers. To control the surface properties of polymers, such as surface resistivity (static or antistatic surfaces), wettability, printability, adhesion, biocompatibility, and lubrication

Correspondence to: A. V. Machado (avm@dep.uminho.pt).

Journal of Applied Polymer Science, Vol. 112, 1595-1600 (2009) (c) 2009 Wiley Periodicals, Inc. (slip), is of critical importance for the use of these materials in a wide range of industrial applications.

To reduce or even eliminate these problems, antistatic additives have been used to modify the electrical properties of the product, reducing the electric resistance of its surface values allowing quick dissipation of the electrostatic charge. ${ }^{1,2}$ Antistatic additives can be internal (added to a polymer during processing) or external (applied to the surface of the product after processing by spraying or wiping). ${ }^{2}$ External antistatic can easily migrate into the polymer.

Although quick to apply and requiring low dosage levels, they are not suitable for high-quality applications, as they can cause prints and sealing problems because of a nonuniform coating. Due to these characteristics, internal antistatic agents are preferred.

Internal antistatic agents can act in two ways: building up a conductive path to bring charged particles into contact and as a lubricant or mold-release agent in the postextrusion process. Because of the incompatibility with the polymer, the additive migrates to the surface of the polymer building a uniform layer. The hydrophilic end projects out of the polymer, the lipophilic end anchors in the polymer. $^{2}$ The protruding hydrophilic end absorbs 
moisture from the surrounding air and builds up a conductive path, reducing the surface resistivity. The rate of migration is dependent on a number of factors, such as, compatibility between the antistatic additives and polymer, polymer crystallinity, total amount of polymer additives, concentration of antistatic, and temperature.

Most of the studies on antistatic additives that have been carried out deal with the polyolefin to be used in the packaging application. ${ }^{3-6}$ Even though, these additives have been widely used in noncrosslinked polyethylene foam for industrial applications, their performance is not yet fully understood and not even systematically studied, essentially due to the peculiarity of its expanded nature. Thus, up to our knowledge, there is a lack of scientific publications on the subject.

The aim of this study was to select the antistatic agent with the best performance that does not compromise the mechanical properties of the final product. Thus, a comparative study was performed with different active antistatic agents, Atmer 7325, Atmer 7105, and Atmer 190, along 6 months of time span in a real life environment. ${ }^{7,8}$

The efficiency of these antistatic agents was assessed by measurements of the surface resistivity and charge decay following the standard procedures.

\section{EXPERIMENTAL}

\section{Materials and chemicals}

A general purpose low density polyethylene (LDPE, Sabic 2102TX00, MFI $=1,9 \mathrm{~g} / 10 \mathrm{~min}$ at $190^{\circ} \mathrm{C} / 2.16$ $\mathrm{kg}$, density $921 \mathrm{~kg} / \mathrm{m}^{3}$ ) was supplied by Sabic. Glycerol monostearate (GMS - Atmer 129) by Ciba, talc micronizate from Ciba, and isobutane gas was purchased from Repsol, and used as received. The antistatic additives, alkoxylated amine (Atmer 7325 and 7105) and alkyl sulfonate (Atmer 190), were supplied by Ciba as masterbatches.

Toluene and 2-propanol (Merck) were of analytical grade: the internal standard (IS) was 1-octadecanol of analytical and high purity grade (Fluka).

\section{Compounding}

LDPE foams were prepared using LDPE, blowing agent (isobutane gas with purity higher than 95\%), nucleating agent (masterbatch of $50 \%$ talc micronizate dispersed in LDPE), and regulator of diffusivity gas agent (Atmer 129). Only the antistatic additives changed (Atmer 7325, 7105, and 190) during preparation. Because the antistatic was a masterbatch consisting of an antistatic agent in low density polyethylene carrier, the amount of antistatic added was $1 \mathrm{wt} \%$ of active antistatic agent in the masterbach. All foams were prepared in an industrial single screw extruder (Myung-II MI-PE 170, L/D = 55) under the same experimental conditions. The barrel set temperature was $200^{\circ} \mathrm{C}$, the screw speed was 21 $\mathrm{rpm}$, and the flow rate was $350 \mathrm{~kg} / \mathrm{h}$. A control sample was prepared using the same experimental procedure but without any antistatic.

After production, all LDPE foams were stored for 180 days under controlled conditions (temperature and humidity of $10-20^{\circ} \mathrm{C}$ and $43 \%$, respectively). The samples were characterized by measuring the residual amount of antistatic and electrical and mechanical properties along this time period.

\section{Quantification of antistatic additives}

The analytical procedure used to quantify the antistatic additives involved three steps: (a) extraction, (b) standard solutions preparation to obtain the calibration curve, and (c) analysis by gas chromatography.

\section{(a) Extraction}

Each LDPE foam $(2 \mathrm{~g})$ was placed in a $250-\mathrm{mL}$ roundbottomed boiling flask with $50 \mathrm{~mL}$ of toluene. The mixture was refluxed for $45 \mathrm{~min}$ at $130^{\circ} \mathrm{C}$. After cooling down at room temperature, 2-propanol was slowly added to precipitate the polymer. The solution was filtered by vacuum and the supernatant liquid evaporated till dryness. The residue obtained was transferred to a volumetric flask and $1 \mathrm{~mL}$ of the IS solution (100 $\mathrm{mg}$ of 1-octadecanol in $100 \mathrm{~mL}$ toluene) was added. Toluene was added to perform a $10 \mathrm{~mL}$ solution.

\section{(b) Preparation of standard solutions to obtain the calibration curve}

The same procedure described in (a) was applied to each masterbatch (with Atmer 7325, 7105, and 190). Dilution of the stock solution was performed with toluene to obtain six standard solutions with different and well known concentrations of each antistatic additive. $1 \mathrm{~mL}$ of the IS solution (100 $\mathrm{mg}$ of 1-octadecanol in $100 \mathrm{~mL}$ toluene) was added to each standard. Toluene was added to perform a $10 \mathrm{~mL}$ solution.

\section{(c) Gas chromatography analysis}

All the samples resulting from steps (a) and (b) were analyzed in triplicate by gas chromatography (GC) using a Chrompack 9001 gas chromatograph equipped with FID detector at $350^{\circ} \mathrm{C}$. Two microliters of each sample were injected in the split-less mode at $250^{\circ} \mathrm{C}$ with a Hamilton syringe HM147000. The GC capillary column was a $15 \mathrm{~m}$ and $0.249 \mathrm{~mm}$ 
I.D. durabond DB-1HT (J and W Scientific) with $100 \%$ based dimethylpolysiloxane stationary phase of $0.10 \mu \mathrm{m}$ thickness. Nitrogen (purity $99.9995 \%$ ) was used as carrier gas. Temperature program: 1 min at $90^{\circ} \mathrm{C}$ then programmed at $10^{\circ} \mathrm{C} / \mathrm{min}$ up to $370^{\circ} \mathrm{C}$ and hold for $50 \mathrm{~min}$.

\section{Density}

This measurement followed the ASTM D 3575 - 00. It covers the determination of foam density by calculation from the mass and volume of a regularly shaped specimen. A representative test specimen of regular shape, not less than $16 \mathrm{~cm}^{3}$ was cut from the sample to be tested. Only a single specimen was tested for each sample. The specimen was weighted on graduated scale to permit weighting within $\pm 1 \%$ of the mass to be measured.

\section{Electrical characterization}

Two tests were carried out to evaluate the electrical performance of the materials. First, the surface resistivity of the samples was obtained by measuring the characteristic I-V curves at room temperature with a Keithley 6487 picoammeter/Voltage source. Contact electrodes were deposited, either by thermal evaporation of aluminum or by silver painting, where the probe tips touched the sample. The current and voltage were measured, and the surface resistivity (Ohm per square) was calculated taken into account the geometrical factors. For each sample, the measurement was performed three times and the final value was taken as the average of the different results obtained. The error associated to this measurement was $\pm 3 \%$.

A decay-time experiment was also performed in the different samples. This test allows characterizing the performance of a given material in the dissipation of $99 \%$ of the charge accumulated, once it was subjected to a $5 \mathrm{kV}$ voltage. The discharge voltagetime graph was obtained making use of a sample holder within a Faraday cage, a variable high voltage source and an Agilent 34401A digital multimeter. The samples with a diameter of $100 \mathrm{~cm}$ were previously conditioned for $48 \mathrm{~h}$ in an atmosphere with relative humidity of $12 \pm 3 \%$. The performance of each sample was measured by comparing the time at which de potential dropped to $50 \pm 5 \mathrm{~V}$. The error associated to this measurement was $\pm 10 \%$.

\section{Mechanical characterization}

Compression set under constant deflection

This test was performed according to a standard issued under the fixed designation D 3575; covering the deflection of the foam specimen under a compressive force and under specified conditions of time and temperature, then measuring the effect on the thickness of the specimen after releasing the compressive force. The compression device, consisting of two or more flat plates arranged to have the plates parallel to each other by bolts or clamps. The space between the plates is adjustable to the required deflection thickness by means of spacers. The test was performed at $23 \pm 2^{\circ} \mathrm{C}$ and in an atmosphere of $50 \pm 5 \%$ relative humidity. Specimens with parallel top and bottom surfaces, essentially perpendicular sides and of $50 \times 50 \mathrm{~mm}$ were plied up, without the use of an adhesive, to produce a total thickness of $25 \mathrm{~mm}$. Once the original thickness $\left(t_{o}\right)$ was measured, the test specimen was placed in the apparatus and compressively deflected to $50 \%$ of its thickness. The test specimen remained deflected in the apparatus for $22 \mathrm{~h}$. After this period of time, it was removed from the test apparatus and after 24 $\mathrm{h}$ of recovery the final thickness $\left(t_{f}\right)$ was measured. The constant deflection compression set was expressed as a percentage of the original thickness. Four specimens for each sample were tested.

\section{Compression deflection}

This test method covers the measurement of the force necessary to produce a $25 \%$ compression over the entire top area of the foam specimen. The apparatus provides a flat compression foot, larger than the specimen to be tested, connected to a force-measuring device, and mounted in a manner such that the specimen can be deflected (compressed) at a speed of $0.2-4 \mathrm{~mm} / \mathrm{s}$.

The test specimen should be a right cylinder with parallel top and bottom surfaces. The thickness shall not be larger than $75 \%$ of the minimum top dimension. Specimens with a minimum area of $2500 \mathrm{~mm}^{2}$ were plied up, without the use of cement, to a minimum of $25 \mathrm{~mm}$. Three specimens for each sample were tested. The specimen was centered in the line of the axial load on the supporting plate of the apparatus. The compression foot was brought into contact with the specimen, and the thickness was determined after applying a total pretest-pressure of $190 \pm 50 \mathrm{~Pa}$ to the specimen. The reading of the load was taken immediately after compressing the specimen $25 \pm 0.5 \%$ of its thickness at $12.5 \mathrm{~mm} /$ min. The compression deflection force per unit of specimen area was calculated.

\section{RESULTS AND DISCUSSION}

\section{Gas chromatography analysis}

The calibration curve was performed taking into account the IS method. The ratio between the antistatic peak area and the IS peak area were plotted 


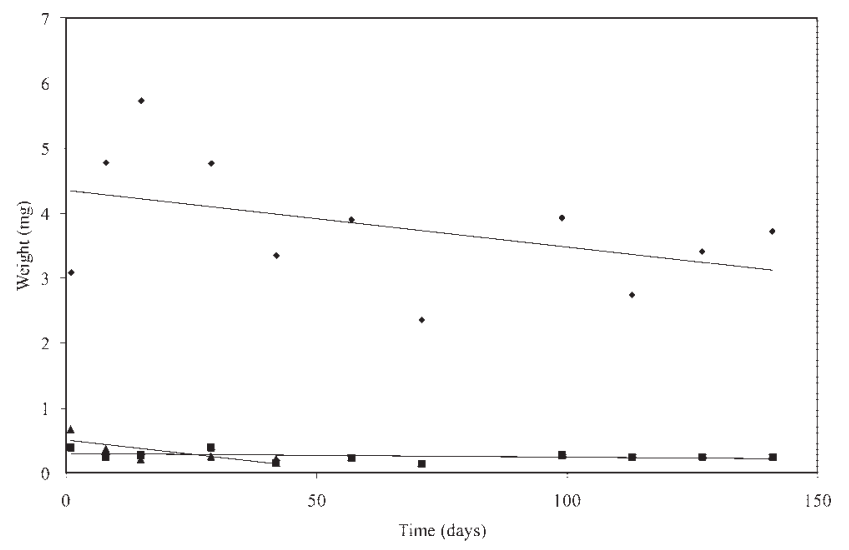

Figure 1 Antistatic weight along storage time: $(\diamond)$ Atmer 7325, (ם) Atmer 7105, and ( $\mathbf{\Delta})$ Atmer 190.

versus the antistatic concentration. The calibration curves obtained were linear from the limit of detection and in agreement with other methods published..$^{9-11}$ GC experiments were carried out to determine the amount of antistatic additives that might migrate during storage. After analysis by gas chromatography in triplicate each extracted sample resulting from LDPE foams, the antistatic additive concentration was determined by interpolation from the corresponding calibration curve. The experimental error associated with the determination of antistatic agent was typically \pm 0.03 mg. The results obtained (Fig. 1) show that the antistatic additives have different behavior. As it can be seen, the antistatic content along storage time depends on the antistatic used, following, in a general way, a linear behavior. The slope obtained is proportional to the migration rate of these additives, ${ }^{12}$ being 6, 10, and $95 \mathrm{mg} /$ day for Atmer 190, 7325 , and 7105, respectively. It can be observed that, while the migration rate of Atmer 190 and 7325 is similar, for Atmer 7105 is $\sim 10$ times higher.

Because the rate of migration depends on a number of factors such as, compatibility, crystallinity, formulation, and temperature, the different behavior in migration rate observed in this study might be mainly related with compatibility.

\section{Density}

As expected, the values of the density of the several foams (Table I) are much lower than for LDPE (921 $\mathrm{kg} / \mathrm{m}^{3}$ ). On the other hand, the values are very similar among them, and both the addition of the regulator of diffusivity gas agent (Atmer 129) and any of the antistatic do not affect the foam density.
TABLE I

Foams Density

\begin{tabular}{lc}
\hline \multicolumn{1}{c}{ Antistatic } & Density $\left(\mathrm{kg} / \mathrm{m}^{3}\right)$ \\
\hline Atmer $129(\mathrm{GMS})$ & 27.2 \\
Atmer 7325 & 28.8 \\
Atmer 7105 & 27.5 \\
Atmer 190 & 29.9 \\
\hline
\end{tabular}

\section{Electrical characterization}

\section{Surface resistance}

Figure 2 shows the time evolution of the surface resistance of each foam studied in this work. The results illustrate that within the 6 months under study all foams containing additive retained the antistatic properties. This fact is in agreement with the chromatography results, as it was observed that the amount of antistatic added to the samples was not completely released during the test period.

On the other hand, the control sample shows an increase of the surface resistivity to values larger than $1 \times 10^{12} \mathrm{Ohm}$ per square, which is typical of insulating materials. This sample shows antistatic properties during $\sim 4$ months. This fact is due to the use of additives for gas diffusion control during the production of the foam, which also act as a short term antistatic agent.

Among the samples with additives, the ones with amina as an active agent (Atmer 7325 and Atmer 7105) show a slightly better antistatic performance than the one with ionic agent (Atmer 190). All these samples show two well defined regions (Fig. 2): a decrease of the surface resistivity during the first month, and a stable behavior for the rest of the test period. This behavior can be related to the results of chromatography, which show a decrease in the amount of antistatic agent due to migration

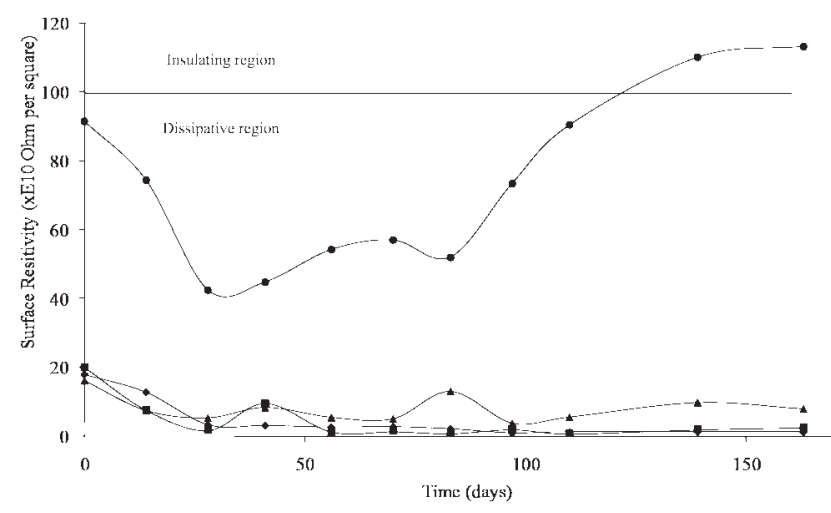

Figure 2 Surface resistivity along 6 months: (O) control

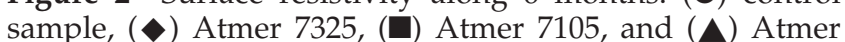
190. 


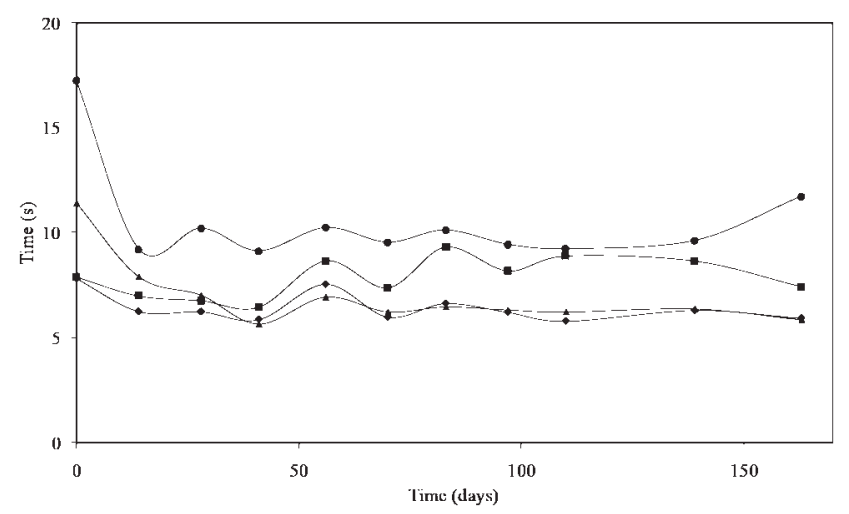

Figure 3 Charge dissipation time along 6 months:

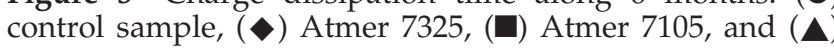
Atmer 190.

processes. Low migration rates will imply increase of the resistivity due to decreasing concentration of antistatic at the surface. Fast migration rates, on the other hand, will imply overall shorter period of antistatic performance of the material due to antistatic dissipation.

\section{Decay rate}

Figure 3 shows the results of the decay-rate measurements for all the samples along the 6 months under test. Each point represents the time needed for the dissipation of $90 \%$ of the $5 \mathrm{kV}$ voltage applied to each sample. The values of the charge dissipation time of the foams with different additives are in the range of $6-13 \mathrm{~s}$. The optimum value of $2 \mathrm{~s}$ was never accomplished.

The dissipation time decreases in all samples during the first 2 weeks after being produced. No significant variations of the performance were observed after this time. The exception to this behavior was the control sample: in this sample an increase of the charge dissipation time is observed, that is correlated with the increase of surface resistivity to values characteristic of insulating materials (Fig. 2). This effect is also related to the use of additives for gas diffusion control, acting as short term antistatic agents.

The sample with ionic (Atmer 190) and amina (Atmer 7325) active agent were the ones with faster dissipation times. Previous studies concluded that the diffusion of additives in crystalline polymers is more difficult than in semicrystalline or amorphous polymers, once that diffusion occurs mainly in the amorphous regions. ${ }^{13,14}$ In foams, diffusion is further hindered by cell porosity, as the additives must migrate by the cell walls towards the material surface. The main issues influencing diffusion of antistatic agents are the length of the hydrocarbon chain and the interaction between the antistatic and the polymeric matrix.

The samples Atmer 7105 showed in all tests worse results than the sample Atmer 7325 . The fact that this additive has a concentration $\sim 10$ times larger than the Atmer 7105 should be the reason for this difference.

Even though ionic active agents are not recommended for polyethylene due to the possibility of thermal degradation, the maximum temperatures used during processing $\left(\sim 170^{\circ} \mathrm{C}\right)$ did not degraded the sodium alkylsulphonate, once the morphological, mechanical, and electrical performance of the Atmer 190 was always appropriate.

\section{Mechanical characterization}

\section{Compression set under constant deflection}

The value of the compression coefficient depends on the recovery of the initial thickness: it is low when the final thickness is approximately equal to the initial thickness. The values obtained for this test along the time are presented in Figure 4, being the experimental error typically $\pm 2 \%$. The control sample shows the lower values of compression coefficient. Samples containing antistatic agents show higher values, being quite similar among then. However, the antistatic that exhibits the better performance is the Atmer7105: it shows the lowest values along the time, the values being nevertheless close to the ones obtained for Atmer 129.

\section{Compression deflection}

Figure 5 depicts the values of compression deflection obtained for the several foams along the time. The experimental error associated was typically $\pm 3 \%$, as estimated from different measurements carried out. The mechanical behavior of the several samples

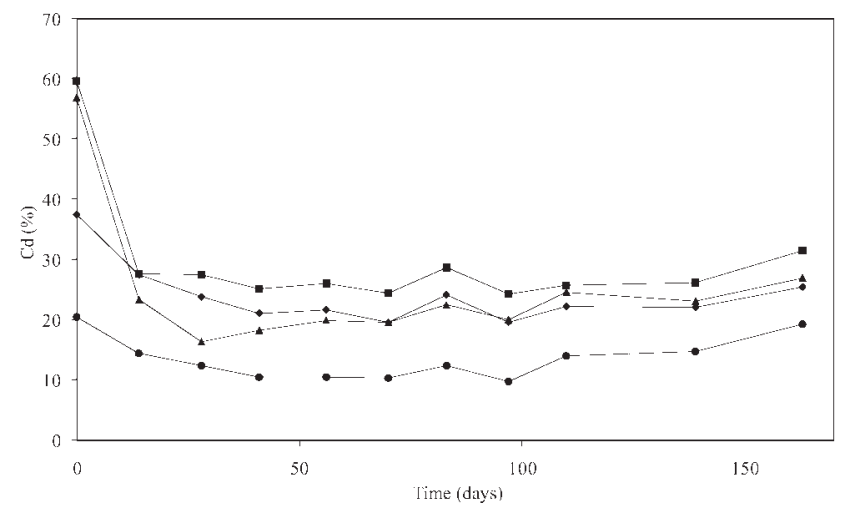

Figure 4 Evolution of the compression coefficient $\left(C_{d}\right)$ along the time: $(\bullet)$ control sample, $(\diamond)$ Atmer 7325 , (ם) Atmer 7105, and (A) Atmer 190. 


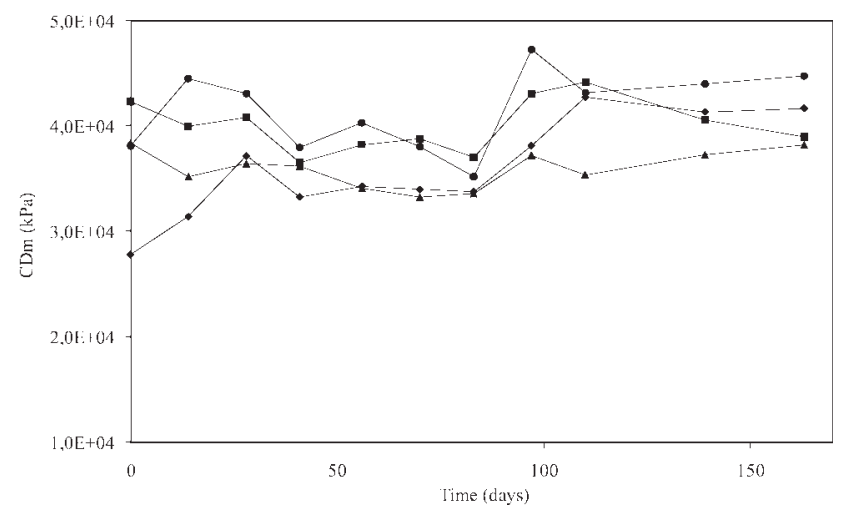

Figure 5 Evolution of the compression deflection along the time: $(\bullet)$ control sample, $(\bullet)$ Atmer 7325, ( $\square$ ) Atmer 7105, and (A) Atmer 190.

along the time is quite similar. Nevertheless, the sample that has the best performance is the foam with antistatic Atmer 7105. The results of mechanical properties obtained along the time show that the addition of an antistatic agent does not significantly affects the mechanical behavior, that is, they do not affect the mechanical performance of the final product.

\section{CONCLUSIONS}

Different antistatic additives were used in the production of LDPE foams. During this study, samples were stored under industrial conditions and the efficiency of antistatic agents was assessed by measurement of the surface resistivity and charge decay, following standard procedures. The migration rate was monitored by gas chromatography and the mechanical performance by compression tests.
This study demonstrates that the migration of antistatic agents in LDPE foams is in general low enough, which allows to maintain the antistatic performance for periods larger than 6 months.

The tests performed during this study revealed that the desired low surface resistance and required low static decay time could be achieved with all the antistatic used. Moreover, the additives with amina (Atmer 7105 and 7325) as an active agent showed slightly better antistatic performance than with the ionic agent (Atmer 190).

\section{References}

1. Drake, N. Polymeric Materials for Electrostatic Applications; Rapra Technology Ltd: United Kingdom, 1996.

2. Fink, H. W. In Plastics Additives Handbook; Gatchter, R.; Muller, H., Eds.; Carl Hanser Verlag: Munich, 1985.

3. Dudler, V.; Grob, M. C.; Merian, D. Polym Degrad Stab 2000, $68,373$.

4. Hausmann, K. Plast Addit Compound 2007, 9, 40.

5. Williams, J. B.; Geick, K. S.; Falter, J. A.; Hall, L. K. J Vinyl Addit Technol 2004, 1, 282.

6. Maki, N.; Nakano, S.; Sasaki, H. Packag Technol Sci 2004, 17, 249.

7. Marvin, R. U.S. Pat. 5,024,792 (1991).

8. Marvin, R.; Joseph, R. U.S. Pat. 5,025,922 (1991).

9. Miller, J. C.; Miller, J. N. Statistics for Analytical Chemistry; Prentice Hall: Great Britain, 1993.

10. Brede, C.; Skjevrak, I.; Herikstad, H. J Chromatogr A 2003, 983, 35.

11. Marcato, B.; Cechin, G. J Chromatogr A 1996, 730, 83.

12. Sakhalkar, S.; Walters, K.; Hirt, D.; Miranda, N.; Roberts, W. J Plast Film Shtg 2002, 18, 33.

13. Inata, H.; Maki, I.; Ishikawa, T.; Takeda, K. J Appl Polym Sci 2006, 99, 2152.

14. Hsu, Y. Y.; Gresser, J. D.; Trantolo, D. J.; Lyons, C. M.; Gangadharam, P. R. J.; Wise, D. L. J Biomed Mater Res 1997, 35, 107. 